\title{
Likelihood-based Tests for Detecting Circadian Rhythmicity and Differential Circadian Patterns in Transcriptomic Applications
}

\author{
Haocheng Ding, ${ }^{1}$ Lingsong Meng, ${ }^{1}$ Andrew C. Liu ${ }^{2}$ Michelle L. Gumz, ${ }^{3}$ \\ Andrew J. Bryant, ${ }^{4}$ Colleen A. Mcclung, ${ }^{5}$ George C. Tseng, ${ }^{6}$ Karyn A. Esser ${ }^{2}$ \\ and Zhiguang Huo ${ }^{1, *}$
}

\footnotetext{
${ }^{1}$ Department of Biostatistics, University of Florida, 2004 Mowry Rd, 32603, FL, USA, ${ }^{2}$ Department of Physiology and Functional Genomics, University of Florida, 1600 SW Archer Rd, 32603, FL, USA, ${ }^{3}$ Division of Nephrology, Hypertension, and Renal Transplantation, Department of Medicine, University of Florida, 1600 SW Archer Rd, 32603, FL, USA, ${ }^{4}$ Division of Pulmonary, Critical Care, and Sleep Medicine, Department of Medicine, University of Florida, 1600 SW Archer Rd, 32603, FL, USA, ${ }^{5}$ Translational Neuroscience Program, Department of Psychiatry, Center for Neuroscience, University of Pittsburgh, 3811 O'Hara Street, 15213, PA, USA and ${ }^{6}$ Department of Biostatistics, University of Pittsburgh, 130 De Soto Street, 15261, PA, USA

*Corresponding author. zhuo@ufl.edu

FOR PUBLISHER ONLY Received on Date Month Year; revised on Date Month Year; accepted on Date Month Year
}

\begin{abstract}
Circadian rhythmicity in transcriptomic profiles has been shown in many physiological processes, and the disruption of circadian patterns has been founded to associate with several diseases. In this paper, we developed a series of likelihood-based methods to detect (i) circadian rhythmicity (denoted as LR_rhythmicity) and (ii) differential circadian patterns comparing two experimental conditions (denoted as LR_diff). In terms of circadian rhythmicity detection, we demonstrated that our proposed LR_rhythmicity could better control the type I error rate compared to existing methods under a wide variety of simulation settings. In terms of differential circadian patterns, we developed methods in detecting differential amplitude, differential phase, differential basal level, and differential fit, which also successfully controlled the type I error rate. In addition, we demonstrated that the proposed LR_diff could achieve higher statistical power in detecting differential fit, compared to existing methods. The superior performance of LR_rhythmicity and LR_diff was demonstrated in two real data applications, including a brain aging data (gene expression microarray data of human postmortem brain) and a time-restricted feeding data (RNA sequencing data of human skeletal muscles). An R package for our methods is publicly available on GitHub https://github.com/diffCircadian/diffCircadian.
\end{abstract}

Key words: circadian rhythmicity, differential circadian analysis, gene expression, likelihood-based test, R package, comparison study

\section{Introduction}

Circadian rhythms are an endogenous $\sim 24$ hours cycle of behavior and physiology including sleep-wake cycles, body temperature, and melatonin $[1,2,3,4]$. Underlying circadian rhythms is the clock mechanism that is found in virtually all cells of body. This mechanism is defined by a transcriptional-translational feedback loop involving a set of core clock genes $[5,6]$, including $C L O C K$, $B M A L 1$, period family (PER1, PER2, PER3), and

(C) The Author 2019. Published by Oxford University Press. All rights reserved. For permissions, please e-mail: 
cryptochrome family ( $C R Y 1, C R Y 2)$. Beyond the core clock mechanism, genome-wide transcriptomic studies have uncovered circadian genes expression patterns in many tissues, including postmodern brain $[7,8]$, skeletal muscle [9], liver [10], and blood [11]. Zhang et al. [12] and Ruben et al. [13] conducted genome-wide circadian analyses using transcriptomic data of 12 unique mouse organs and 13 unique human organs, respectively, and showed that the profiles of circadian gene expression were tissue specific. It is now recognized from studies in humans and rodents that disruption in clock and circadian gene expression are linked to diseases including type II diabetes [14], sleep [11], major depression disease [15], aging [7], schizophrenia [8], and Alzheimer's disease [16].

In the literature, several algorithms have been developed to detect circadian rhythmicity, including Ftest via cosinor-based rhythmometry [17], Lomb-Scargle periodograms [18], COSOPT [19], ARSER [20], RAIN [21], JTK CYCLE [22], and MetaCycle [23]. These algorithms were widely applied in transcriptomic studies, and the comparisons of these algorithm have been evaluated in several review studies $[24,25,26]$. Though promising, concerns have been raised [25] that the p-values generated by many of these existing methods may not be correct (i.e., do not follow a uniform distribution [i.e., $\mathrm{U}(0,1)$ ] under the null), implying a potential inflated or deflated type I error rate.

Another increasingly important research question is to identify differential circadian patterns associated with different experimental conditions [27, 28, 11]. Figure 1 shows 4 types of differential circadian patterns identified in our brain aging data application (see Section 4.1 for details), among which 31 subjects were from the young group (age $\leq 40$ years), and 37 subjects were from the old group (age $>60$ years). Gene CIART in Figure 1a shows the differential amplitude, where the amplitude in the young group is larger than the old group; Gene PER2 in Figure 1b shows the differential phase, where the phases in young and old groups are different; Gene TRIB2 in Figure 1c shows the differential basal level, where the basal level in the young group is higher than the old group; Gene MYO5A in Figure 1d shows the differential fit, where there exists a good circadian rhythmicity fit in the young group, but not in the old group. The definition of amplitude, phase, and basal level is illustrated in Figure 2. The traditional approach to compare circadian rhythmicity between two experimental conditions is to adopt a hard threshold (e.g., $p \leq 0.01$ ) as the significance cutoff, and then declare deferential circadian rhythmicity if a gene is significant in only one condition [11, 29]. Though straightforward, this approach may fail under the following two scenarios. Scenario (i): gene PER2 in Figure $1 \mathrm{~b}$ is showing significant circadian rhythmicity in both the young group $\left(p=1.60 \times 10^{-4}\right)$ and the old group $\left(p=7.37 \times 10^{-5}\right)$, and thus did not satisfy the definition of differential circadian pattern. However, Figure 1b shows a clear phase difference comparing young and old groups, and the underlying differential phase p-value using our proposed method was $5.43 \times 10^{-5}$. Scenario (ii): gene EEF2K had a circadian p-value 0.0096 in young group, and a p-value 0.0305 in the old group. Though this gene

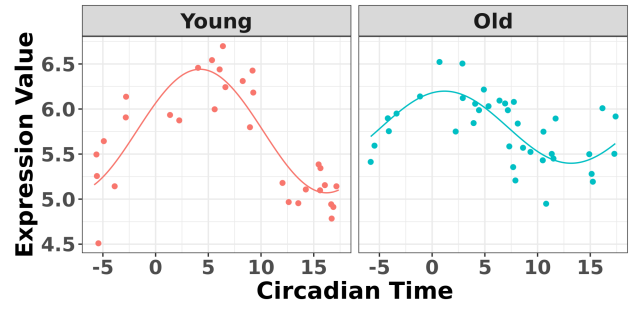

(a) Differential amplitude for CIART $(p=0.008)$

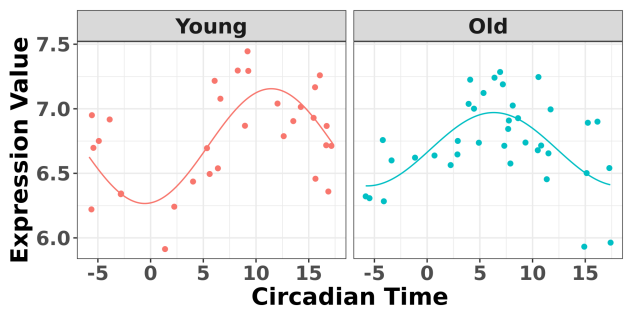

(b) Differential phase for PER2 $\left(p=5.43 \times 10^{-5}\right)$

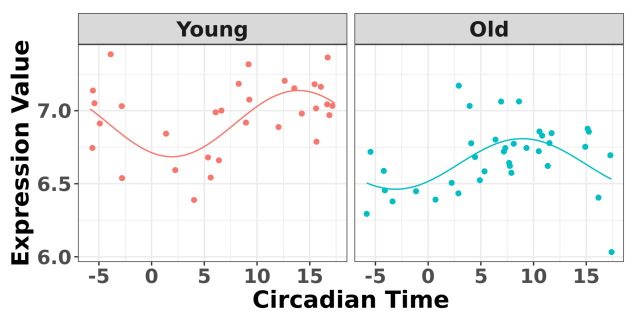

(c) Differential basal level for TRIB2 $(p=2.88 \times$ $\left.10^{-6}\right)$

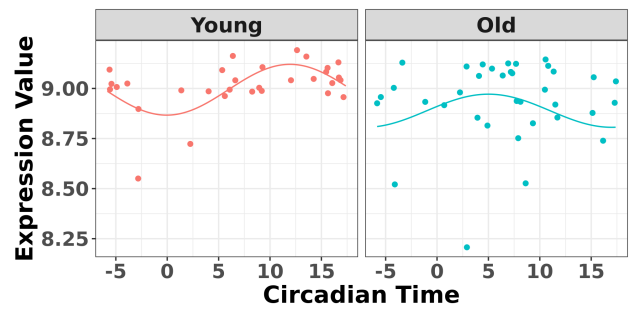

(d) Differential fit for MYO5A $\left(p=4.34 \times 10^{-8}\right)$

Fig. 1. The most significant genes showing four types of differential circadian patterns from the brain aging data.

satisfied this definition of differential circadian pattern using $p \leq 0.01$ as the significance criteria, the rhythmicity p-values under both conditions were close to 0.01. In fact, the resulting differential fit p-value using our proposed method was 0.709 , indicating EEF2K was not showing differential circadian pattern comparing the young group and the old group.

In the literature, some methods have been developed to identify genes showing differential circadian patterns. Chen et al. [7] developed a permutation test to quantify the statistical significance of these 4 types of differential circadian patterns. However, the nonparametric permutation test could suffer from low 
p-value precision and heavy computational burdens. DODR [30] and LimoRhyde [31] were developed to examine the hypothesis that the circadian rhythmicity across two conditions are identical, but they failed to further categorize different subclasses of differential circadian patterns illustrated in Figure 1. More recently, circaCompare [32] was developed to detect differential amplitude, differential phase, and differential basal level using non-linear least square methods, but it could not characterize differential fit. To our knowledge, there is still a lack of unified parametric method that could identify all 4 differential circadian patterns simultaneously. In addition, the performance of these existing methods has not been systematically evaluated.

In the statistics field, likelihood-based methods enjoyed tremendous popularity for its simplicity when testing single parameter and its flexibility to extend to test multiple parameters or complex models. In addition, the testing procedures based on the likelihood-based methods are generally considered as asymptotically the most efficient. However, this concept has not been fully developed in the field of circadian analysis. To close these research gaps, and to fully incorporate the merit of likelihood-based approaches, we propose a series of likelihood-based methods to detect circadian rhythmicity (within one condition) as well as differential circadian patterns (comparing two conditions). The contribution and novelty of this paper includes: (i) systematically evaluated the accuracy of p-values in detecting circadian rhythmicity of our likelihood-based methods and other existing methods. (ii) the first to propose likelihood-based methods to identify all 4 types of differential circadian patterns; (iii) systemically evaluated our likelihood-based methods in detecting differential circadian patterns, and compared with existing methods in terms of the correctness of p-value and statistical power; (vi) implemented our proposed methods in $\mathrm{R}$ software package, which has been made publicly available on GitHub.

\section{Method}

We developed likelihood-based methods for (i) circadian rhythmicity detection within one experimental condition and (ii) differential circadian pattern analysis comparing two experimental conditions. The statistical inference of these methods were based on the Wald statistics and the likelihood ratio statistics. Since the accurate inference of the likelihood-based methods required large sample size, we also employed finite sample corrections to improve the performance under small sample sizes.

Notations for a sinusoidal wave fitting

Our methods assume that the relationship between the gene expression level and the circadian time fits a sinusoidal wave curve. As illustrated in Figure 2, denote $y$ as the expression value for a gene; $t$ as the circadian time; $C$ as the basal level (vertical shift of the sinusoidal wave baseline from 0); $A$ as the amplitude. $\omega$ is the frequency of the sinusoidal wave, where $\omega=\frac{2 \pi}{\text { Period }}$. Without loss

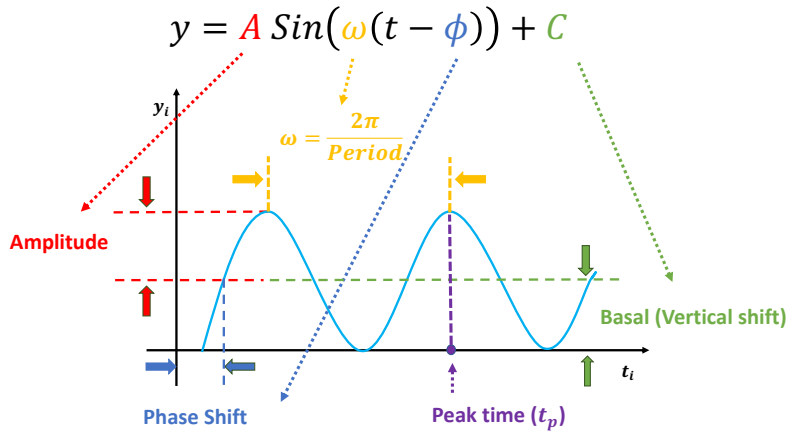

Fig. 2. Illustration of a sinusoidal wave fitting and its related terminologies.

of generality, we set period $=24$ hours to mimic the diurnal period. $\phi$ is the phase of the sinusoidal wave curve. Whenever there is no ambiguity, we will omit the unit "hours" in period, phase, and other related quantities. Due to the periodicity of a sinusoidal wave, $\left(\phi_{1}, \phi_{2}\right)$ are not identifiable when $\phi_{1}=\phi_{2}+24$. Therefore, we will restrict $\phi \in[-6,18) . \phi$ may be difficult to read from a sinusoidal wave (See Figure 2), and a closely related quantify is the peak time $t_{P}$. The connection between $\phi$ and $t_{P}$ is that $\phi+t_{P}=6 \pm 24 N$, where $N$ is an arbitrary natural number.

\section{Circadian Rhythmicity Detection}

In this section, we develop likelihood-based methods to test the existence of a circadian rhythmicity within one experimental condition. Denote $y_{i}$ is the expression value of one gene for subject $i(1 \leq i \leq n)$, where $n$ is the total number of subjects. $t_{i}$ is the circadian time for subject $i$. We assume:

$$
y_{i}=A \sin \left(\omega\left(t_{i}+\phi\right)\right)+C+\varepsilon_{i},
$$

where $\varepsilon_{i}$ is the error term for subject $i$; we assume $\varepsilon_{i}$ 's are identically and independently distributed (i.e., iid) and $\varepsilon_{i} \sim N\left(0, \sigma^{2}\right)$, where $\sigma$ is the noise level. To benchmark the goodness of sinusoidal wave fitting, we define the coefficient of determination $R^{2}=1-\frac{R S S}{T S S}$, where $R S S=\sum_{i=1}^{n}\left(y_{i}-\hat{y}_{i}\right)^{2}, T S S=\sum_{i=1}^{n}\left(y_{i}-\bar{y}\right)^{2}$, $\hat{y}_{i}=\hat{A} \sin \left(\omega\left(t_{i}+\hat{\phi}\right)\right)+\hat{C}, \bar{y}=\sum_{i} y_{i} / n$, with $\hat{A}, \hat{\phi}$, and $\hat{C}$ being the fitted value for $A, \phi$, and $C$ in Equation 1 under least square loss, respectively. $R^{2}$ ranges from 0 to 1 , with 1 indicating perfect sinusoidal wave fitting, and 0 indicating no fitting at all. Based on these assumptions, we derive procedures for testing circadian rhythmicity. For the ease of discussion, we re-write Equation 1 as

$$
y_{i}=E \sin \left(\omega t_{i}\right)+F \cos \left(\omega t_{i}\right)+C+\varepsilon_{i}
$$

where $E=A \cos (\omega \phi)$, and $F=A \sin (\omega \phi)$. The hypothesis setting for testing the existence circadian rhythmicity is $H_{0}: E=F=0$ v.s. $H_{A}: E \neq 0$ or $F \neq 0$. We will derive the Wald statistics and the likelihood ratio statistics to perform hypothesis testing. Since both Wald statistics and likelihood ratio statistics are designed based on large 
sample theories, we will also employ finite sample statistics for these methods.

Likelihood Ratio Test

Based on Equation 2, the likelihood function of all $n$ samples is:

$$
\begin{aligned}
& L\left(E, F, C, \sigma^{2}\right) \\
& =\prod_{i=1}^{n} L_{i}\left(E, F, C, \sigma^{2} \mid y_{i}, t_{i}\right) \\
& =\prod_{i=1}^{n} \frac{1}{\sqrt{2 \pi \sigma^{2}}} \exp \left(-\frac{\left(y_{i}-E \sin \left(\omega t_{i}\right)-F \cos \left(\omega t_{i}\right)-C\right)^{2}}{2 \sigma^{2}}\right)
\end{aligned}
$$

The log-likelihood function is:

$$
\begin{aligned}
& l\left(E, F, C, \sigma^{2}\right) \\
& =\log L\left(E, F, C, \sigma^{2}\right) \\
& =-\frac{n}{2} \log \left(2 \pi \sigma^{2}\right)-\sum_{i=1}^{n} \frac{\left(y_{i}-E \sin \left(\omega t_{i}\right)-F \cos \left(\omega t_{i}\right)-C\right)^{2}}{2 \sigma^{2}}
\end{aligned}
$$

Under $H_{0}, \boldsymbol{\beta}=\boldsymbol{\beta}_{0}=\left(0,0, \hat{C}_{0}, \hat{\sigma}_{0}^{2}\right)^{\top}$, and $l_{0}=$ $l\left(0,0, \hat{C}_{0}, \hat{\sigma}_{0}^{2}\right)$, where $\boldsymbol{\beta}_{0}$ is the least square estimate of Equation 2 under $H_{0}$. Under $H_{A}, \boldsymbol{\beta}=\boldsymbol{\beta}_{A}=$ $\left(\hat{E}_{A}, \hat{F}_{A}, \hat{C}_{A}, \hat{\sigma}_{A}^{2}\right)^{\top}$ and $l_{A}=l\left(\hat{E}_{A}, \hat{F}_{A}, \hat{C}_{A}, \hat{\sigma}_{A}^{2}\right)$, where $\boldsymbol{\beta}_{A}$ is the least square estimate of Equation 2. The likelihood ratio test statistic is: $T^{L R}=-2\left(l_{0}-l_{A}\right)$. Since the degree of freedom is 2 , under $H_{0}, T^{L R} \sim \chi_{2}^{2}$.

Wald Test

The Wald test statistic can be derived as $T^{\text {Wald }}=\left(\boldsymbol{\beta}_{A}-\right.$ $\left.\boldsymbol{\beta}_{0}\right)^{\top} I\left(\boldsymbol{\beta}_{A}\right)\left(\boldsymbol{\beta}_{A}-\boldsymbol{\beta}_{0}\right)$, where $I\left(\boldsymbol{\beta}_{A}\right)$ is Fisher information matrix evaluated at $\boldsymbol{\beta}_{A}$. Under $H_{0}, T^{\text {Wald }} \sim \chi_{2}^{2}$.

Finite Sample Wald/LR Tests

The Wald test and the likelihood ratio test may have inflated type I error when sample size is small since they rely on large sample asymptotic theory. Parker [33] introduced finite sample Wald and likelihood ratio test statistics, which could better control the type I error rate to the nominal level even with small sample sizes. The finite sample Wald statistics $\left(T_{F N}^{W \text { ald }}\right)$ and the finite sample likelihood ratio statistics $\left(T_{F N}^{L R}\right)$ can be derived as the following:

$$
\begin{gathered}
T_{F N}^{W \text { ald }}=\frac{n-k+1}{n r} T^{\text {Wald }} \\
T_{F N}^{L R}=\frac{n-k+1}{r}\left(\exp \left(T^{L R} / n\right)-1\right)
\end{gathered}
$$

Where $k=4$ is total number of parameters and $r=$ 2 is number of parameters of interest. Under the null hypothesis, $T_{F N}^{W \text { ald }} \sim F\left(d f_{1}, d f_{2}\right)$, and $T_{F N}^{L R} \sim F\left(d f_{1}, d f_{2}\right)$, where $d f_{1}=r$ and $d f_{2}=n-k+1$.

F Test

The F-test method to detect the circadian rythmicity has been previously established [17]. F test constructs its test statistic by decomposing total variability into model sum of square, and residual sum of square, which is closely related to our proposed finite sample likelihood method. Thus, we also describe the F-test method in our manuscript, and will draw connection between F-test and our likelihood method.

$$
T^{F}=\frac{(T S S-R S S) / d f_{1}}{R S S / d f_{2}} \sim F\left(d f_{1}, d f_{2}\right),
$$

where residual sum of squares $(R S S)=\sum_{i=1}^{n}\left(y_{i}-\hat{y}_{i}\right)^{2}$, total sum of squares $(T S S)=\sum_{i=1}^{n}\left(y_{i}-\bar{y}\right)^{2}, d f_{1}=2$ and $d f_{2}=n-3$. Under the null hypothesis, $T^{F} \sim F\left(d f_{1}, d f_{2}\right)$.

Other competing methods

We will compare our proposed likelihood-based method to other existing methods, including F-test [17], ARSER [20], Lomb-Scargle periodograms [18], JTK CYCLE [22], RAIN [21], and the permutation test [7]. ARSER, RAIN, and JTK CYCLE have some special requirement for the input circadian time - the input circadian time has to be integer value, and the intervals between two adjacent circadian time points must be the same. Thus, we will accommodate such design in our simulation settings when needed.

\section{Differential Circadian Analysis}

In this section, we develop likelihood-based testing procedures to identify genes showing differential circadian patterns, including (a) differential amplitude, (b) differential phase, (c) differential basal level, and (d) differential fit, as shown in Figure 1.

Denote $y_{1 i}$ as the gene expression value of subject $i(1 \leq$ $\left.i \leq n_{1}\right)$ in experimental condition 1 , where $n_{1}$ is the total number of subjects; $t_{1 i}$ is the circadian time for subject $i$. $y_{2 j}$ is the gene expression value of subject $j\left(1 \leq j \leq n_{2}\right)$ in experimental condition 2, where $n_{2}$ is the total number of subjects; $t_{2 j}$ is the circadian time for subject $j$. Note that $y_{1 i}$ and $y_{2 j}$ are from the same gene, but under different experimental conditions. We assume the following models:

$$
\begin{aligned}
& y_{1 i}=A_{1} \sin \left(\omega\left(t_{1 i}+\phi_{1}\right)\right)+C_{1}+\varepsilon_{1 i} \\
& y_{2 j}=A_{2} \sin \left(\omega\left(t_{2 j}+\phi_{2}\right)\right)+C_{2}+\varepsilon_{2 j}
\end{aligned}
$$

$\varepsilon_{1 i} \sim N\left(0, \sigma_{1}^{2}\right)$ is the error term for subject $i\left(1 \leq i \leq n_{1}\right)$ for experimental condition 1 and $\varepsilon_{2 j} \sim N\left(0, \sigma_{2}^{2}\right)$ is the error term for subject $j\left(1 \leq j \leq n_{2}\right)$ for experimental condition 2. These error terms are assumed to be iid. $A_{1}$, $\phi_{1}, C_{1}$, and $\sigma_{1}^{2}$ are the amplitude, phase, basal level, and noise level for the experimental condition 1 , and $A_{2}, \phi_{2}$, $C_{2}$, and $\sigma_{2}^{2}$ are for experimental condition 2 .

Hypothesis testing framework for differential circadian analysis

Below we state the null hypothesis and the alternative hypothesis for testing these four categories of differential circadian patterns, based on Equation 5.

1. Differential amplitude: $H_{0}: A_{1}=A_{2}=A_{c}$ v.s. $H_{A}$ : $A_{1} \neq A_{2}$.

2. Differential phase: $H_{0}: \phi_{1}=\phi_{2}=\phi_{c}$ v.s. $H_{A}: \phi_{1} \neq$ $\phi_{2}$. 
3. Differential basal level: $H_{0}: C_{1}=C_{2}=C_{c}$ v.s. $H_{A}$ : $C_{1} \neq C_{2}$.

4. Differential fit: $H_{0}: \sigma_{1}^{2}=\sigma_{2}^{2}=\sigma_{c}^{2}$ v.s. $H_{A}: \sigma_{1}^{2} \neq \sigma_{2}^{2}$.

Remark 1 (i) As suggested by Chen et al. [7], the circadian rhythmicity can be characterized by the goodness of fit statistics $R^{2}$. Since it is not easy to derive statistical inference on $R^{2}$, we will use a closely related quantity, $\sigma^{2}$, to quantify the goodness of fit. (ii) The prerequisite for differential amplitude, differential phase, and differential basal level is that there should exist circadian rhythmicity in both conditions under comparisons. Therefore, we suggested users to set $p \leq$ 0.01 or $p \leq 0.05$ from our previous likelihood-based circadian rhythmicity test to ensure the existence of the circadian rhythmicity in both conditions. (iii) The prerequisite for differential fit is that there should exist a circadian rhythmicity in either experimental conditions. We suggested users to set $p \leq 0.01$ or $p \leq 0.05$ from our previous likelihood-based circadian rhythmicity test to ensure such prerequisite.

Likelihood ratio test

Based on Equation 5, the log-likelihood function for $n_{1}+n_{2}$ samples in both experimental conditions is:

$$
\begin{aligned}
& l l\left(A_{1}, \phi_{1}, C_{1}, \sigma_{1}^{2}, A_{2}, \phi_{2}, C_{2}, \sigma_{2}^{2}\right) \\
& =\sum_{i=1}^{n_{1}} l_{i}\left(A_{1}, \phi_{1}, C_{1}, \sigma_{1}^{2}\right)+\sum_{j=1}^{n_{2}} l_{j}\left(A_{2}, \phi_{2}, C_{2}, \sigma_{2}^{2}\right) \\
& =\left(-\frac{n_{1}}{2} \log \sigma_{1}^{2}-\sum_{i=1}^{n_{1}} \frac{\left(y_{i}-A_{1} \sin \left(\omega\left(t_{i}+\phi_{1}\right)\right)-C_{1}\right)^{2}}{2 \sigma_{1}^{2}}\right) \\
& +\left(-\frac{n_{2}}{2} \log \sigma_{2}^{2}-\sum_{j=1}^{n_{2}} \frac{\left(y_{j}-A_{2} \sin \left(\omega\left(t_{j}+\phi_{2}\right)\right)-C_{2}\right)^{2}}{2 \sigma_{2}^{2}}\right)
\end{aligned}
$$

The test statistic is: $D^{L R}=-2\left(l l_{0}-l l_{A}\right)$, where $l l_{0}$ is the $\log$ likelihood under $H_{0}$; and $l l_{A}$ is the log likelihood under $H_{A}$. Here the null can be one of the following: (a) $H_{0}^{(a)}: A_{1}=A_{2}$ for differential amplitude; (b) $H_{0}^{(p)}$ : $\phi_{1}=\phi_{2}$ for differential phase; (c) $H_{0}^{(b)}: C_{1}=C_{2}$ for differential basal level; (d) $H_{0}^{(f)}: \sigma_{1}^{2}=\sigma_{2}^{2}$ for differential fit. For all these null hypotheses, the degree of freedom is 1 , and $D^{L R} \sim \chi_{1}^{2}$ under $H_{0}$. For example when testing different amplitude, under $H_{0}^{(a)}, \hat{A_{1}}=\hat{A_{2}}=\hat{A_{c}}$, $l l_{0}=l l\left(\hat{A_{c}}, \hat{\phi_{1}}, \hat{C_{1}}, \hat{\sigma_{1}}, \hat{A_{c}}, \hat{\phi_{2}}, \hat{C_{2}}, \hat{\sigma_{2}} \mid y\right) ;$ and under $H_{A}^{(a)}$, $\hat{A_{1}} \neq \hat{A_{2}}, l l_{A}=l l\left(\hat{A_{1}}, \hat{\phi_{1}}, \hat{C_{1}}, \hat{\sigma_{1}}, \hat{A_{2}}, \hat{\phi_{2}}, \hat{C_{2}}, \hat{\sigma_{2}} \mid y\right)$.

Wald Test

Denote $\boldsymbol{p}=\left(A_{1}, \phi_{1}, C_{1}, \sigma_{1}^{2}, A_{2}, \phi_{2}, C_{2}, \sigma_{2}^{2}\right)^{\top} \cdot \boldsymbol{p}_{0}$ is $\boldsymbol{p}$ under $H_{0}$, where $H_{0}$ is one of the null hypotheses in Section 2.3.1; $\boldsymbol{p}_{1}$ is $\boldsymbol{p}$ under $H_{A}$, where there is no restriction on $\boldsymbol{p}$. Then the Wald test statistic is $D^{\text {Wald }}=\left(\boldsymbol{p}_{1}-\boldsymbol{p}_{0}\right)^{\top} I\left(\boldsymbol{p}_{1}\right)\left(\boldsymbol{p}_{1}-\right.$ $\left.\boldsymbol{p}_{0}\right)$. Under $H_{0}, D^{\text {Wald }} \sim \chi_{1}^{2}$, where $I\left(\boldsymbol{p}_{1}\right)$ is Fisher information matrix evaluated at $\boldsymbol{p}_{1}$.

Finite Sample Wald/LR Tests

Again, in order to control type I error for small sample sizes, we derive finite sample version of the Wald statsitics and likelihood ratio statistics $D_{F N}^{W a l d}$ and $D_{F N}^{L R}$ by Equations 3 and 4 . Under $H_{0}, D_{F N}^{\text {Wald }} \sim F\left(d f_{1}, d f_{2}\right)$, and $D_{F N}^{L R} \sim F\left(d f_{1}, d f_{2}\right)$, where $d f_{1}=r$ and $d f_{2}=n-k+1$.

Competing methods for differential circadian analysis We will compare the performance of our method with other existing methods, including the permutation test [7], DODR [30], LimoRhyde [31], and circaCompare [32]. We acknowledge that HANOVA, robustDODR and LimoRhyde are designed to detect differential rhythmicity (i.e., whether the circadian rhythmicity across two conditions are identical), and cannot distinguish the 4 subcategories in Figure 1 . Thus we will apply these two methods in detecting differential fit, which is closely related to differential rhythmicity conceptually; circaCompare can examine differential amplitude, differential phase, and differential basal level; while the permutation test as well as our proposed method can examine all four types if differential circadian patterns illustrated in Figure 1.

\section{Simulation}

In terms of circadian rhythmicity detection, we demonstrated that our proposed method correctly controlled the type I error rate to the nominal level, while some of the other methods failed to control the type I error rate. In terms of differential circadian pattern detection, our method still controlled the type I error rate to the nominal level. For differential fit, which is one type of the differential circadian pattern shown in Figure 1d, we demonstrated our method achieved higher statistical power compared to the existing methods.

Simulation for Circadian Rhythmicity Analysis

Simulation Settings

Denote $i(1 \leq i \leq n)$ as the sample index, where $n$ was the total number of samples. The circadian time $t_{i}$ for sample $i$ was generated from uniform distribution $\operatorname{UNIF}(0,24)$. We simulated the gene expression value for sample $i$ using Equation 1.

$$
y_{i}=A \sin \left(\omega\left(t_{i}+\phi\right)\right)+C+\varepsilon_{i} .
$$

Our basic parameter setting for simulation is listed as below. For each gene, the sample size $n$ was set to be 12; the circadian time were sampled every 2 hours (i.e., $\left.t_{1}=1, t_{2}=3, \ldots, t_{12}=23\right)$, such integer circadian time and evenly spaced interval time are required by some other existing methods. Whenever the statistical methods have no such requirement, we sampled circadian time directly from $\operatorname{UNIF}(0,24)$. Amplitude $A$ was fixed at 1 ; phase $\phi$ was generated from $\operatorname{UNIF}(0,24)$. Basal level $\mathrm{C}$ was generated from $\operatorname{UNIF}(0,3)$. Error term $\varepsilon_{i}$ was generated from normal distribution $N\left(0, \sigma^{2}\right)$ where $\sigma^{2}$ was set to be 1 . We simulated $G=10,000$ genes for each simulation, and each simulation was repeated $B=10$ times to increase numbers of replications and to obtain an standard error estimate. To examine whether our method is robust against higher signal-noise ratios, correlated gene structures, and violations of normality distributions, we further simulates the following variations: 
1. Impact of sample sizes. We varied $n=6,12,24,48,96$ while fixing other parameters in the basic parameter setting fixed. Note that when $n>24$, we would allow repetitive circadian time for different samples. For example, when $n=48$, the circadian time sequence would be $t_{1}=1, t_{2}=1, t_{3}=2, \ldots, t_{47}=24$, and $t_{48}=24$.

2. Impact of signal noise ratio. The signal noise ratio is defined as $\frac{A}{\sigma}$. Thus we varied $\sigma=1,2,3$ to mimic varying levels of signal noise ratio, while fixing other parameters in the basic parameter setting.

3. Impact of correlated genes. In transcriptomic data applications, individual genes are can be be correlated. Thus, we simulated the following correlated structure. For every $\mathrm{m}=50$ genes, we simulated

$$
\boldsymbol{y}=A \sin (\omega(\boldsymbol{t}+\phi))+C+\boldsymbol{\varepsilon},
$$

where $\boldsymbol{y}=\left(y_{1}, \ldots, y_{m}\right), \boldsymbol{t}=\left(t_{1}, \ldots, t_{m}\right)$, and $\boldsymbol{\varepsilon}=$ $\left(\varepsilon_{1}, \ldots, \varepsilon_{m}\right)$. In this case, $\boldsymbol{\varepsilon}$ were generated from a multivariate normal distribution $\operatorname{MVN}(0, \boldsymbol{\Sigma})$. And $\boldsymbol{\Sigma} \in \mathbb{R}^{m \times m}$ was the covariance matrix generated from the inverse Wishart distribution $W^{-1}(\boldsymbol{\Phi}, v)$. In order to mimic correlated gene structure, we first designed $\Phi^{\prime}=(1-\rho) I_{m \times m}+\rho J_{m \times m}$, and then standardized $\Phi^{\prime}$ to correlation matrix $\Phi$, where $I_{m \times m}$ was the identify matrix, and $J_{m \times m} \in \mathbb{R}^{m \times m}$ a matrix with all elements 1 . We fixed $v$ to be 60 , and vary $\rho=0,0.25,0.5$.

4. Violation of the Gaussian assumption. Instead of assuming the error term was generated from a standard normal distribution (i.e., $N(0,1)$ ), we generated $\varepsilon_{1} \sim t(3), t(5), t(10), t(\infty)$, where $t(d f)$ is the t-distribution with degree of freedom $d f$. This family of t-distributions represents long tailed error distribution, with smaller $d f$ indicating longer tailed error distribution, and thus larger violation of the normality assumption. When $d f=\infty, t(\infty)$ is the same as $N(0,1)$.

The best performer of the likelihood based methods in detecting circadian rhythmicity

Before comparing with other existing methods, we first evaluated the Type I error rate (nominal $\alpha$ level 5\%) of our proposed four likelihood-based methods in detecting circadian rhythmicity, including Wald test (regular), Wald test (finite sample), likelihood ratio test (regular), likelihood ratio test (finite sample). Since the limiting distribution of both Wald statistics (finite sample) and likelihood ratio statistics (finite sample) follows an $\mathrm{F}$ distribution, we also include the F-test method [17] as benchmark.

Figure S1 showed type I error rates (nominal $\alpha$ level $5 \%$ ) of our proposed four methods and the F-test method. Regardless of the varying sample sizes, the Wald test (finite sample), the likelihood ratio test (finite sample), and the F-test controlled the type I error rate close to the $5 \%$ nominal level, while the Wald test (regular) and the likelihood ratio test (regular) obtained inflated type I error rate. The Wald test (regular) and the likelihood ratio test (regular) had better performance when sample size became larger, which was not unexpected because these asymptotic tests rely on large sample sizes. Remarkably, we observed that the Wald test (finite sample) and the likelihood ratio test (finite sample) achieved almost the same test statistics as the F-test, indicating the finite sample approximation procedure [33] successfully convert our likelihood-based statistics to F statistics.

Similar results was also observed by varying signal noise ratio (Figure S2) and varying the strength of gene correlations (Figure S3). The Wald test (finite sample), the likelihood ratio test (finite sample), and the F-test could better control the type I error rate to the $5 \%$ nominal level compared to the the Wald test (regular) and the likelihood ratio test (regular).

As shown in Figure S4, when we varied the level of normality violation by varying $d f$ of the tdistribution, we observed that all test procedures became more conservative, which is not unexpected because our likelihood-based method assumed Gaussian error in the likelihood construction. In practice, if the residuals (i.e., $y_{i}-\hat{y}_{i}$ ) violated the Gaussian distribution, we would recommend data transformations (e.g., log transformation) to improve normality.

To summarize, the Wald test (finite sample) and the likelihood ratio test (finite sample) are the best performer of our proposed likelihood-based methods in detecting circadian rhythmicity, which could control the type I error rate to the nominal level under the Gaussian assumption. And these two methods are equivalent to the F-test method in terms of the test statistics. Therefore, we will pick up the likelihood ratio test (finite sample) as the representative of our proposed methods in detecting circadian rhythmicity, and we denoted LR_rhythmicity as the short name for this method in all later evaluations.

Type I Error rate comparison with other methods We compared the likelihood-based method (LR_rhythmicity) with other existing methods in detecting circadian rhythmicity, including Lomb-Scargle, JTK, ARSER, Rain, and permutation. We excluded the $\mathrm{F}$ test in our evaluation, since it is essentially the same as LR_rhythmicity. Figure 3 showed type I error rates by varying sample sizes. In general, LR_rhythmicity and the permutation test controlled the type I error rate to the 5\% nominal level, while the other methods had inflated or conservative type I error rate. Similar results was also observed by varying signal noise ratio (Figure S5) and varying the strength of gene correlations (Figure S6). As shown in Figure S7, we observed that a larger violation of normality assumption will lead to more conservative type I error rate for LR_rhythmicity, while the permutation test can still control the type I error rate to the $5 \%$ nominal level.

To summarize, under the Gaussian assumption (i.e., the residual follows normal distribution), only the LR_rhythmicity and the permutation test can achieve nominal type I error rate control (i.e., 5\%).

\section{Power analysis}

For the power analysis, we only examined the method that could successfully control the type I error rate to the $5 \%$ nominal level. Otherwise the power is directly not 


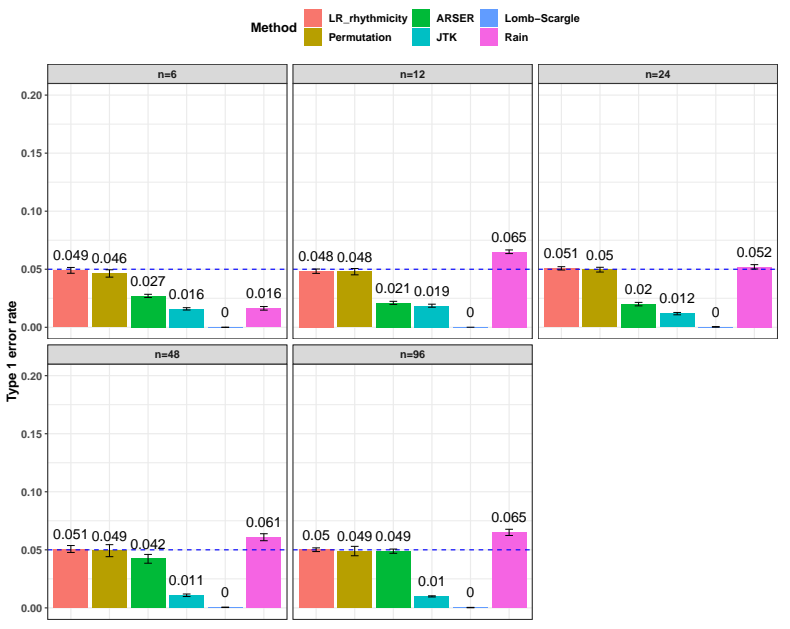

Fig. 3. Type I error rate at nominal $\alpha$ level $5 \%$ for 6 different methods in detecting circadian rhythmicity. The sample sizes were varied at $\mathrm{n}=6,12,24,48$, and 96 . The blue dashed line is the $5 \%$ nominal level. A higher than $5 \%$ blue dashed line bar indicates an inflated type I error rate; a lower than $5 \%$ blue dashed line bar indicates a conservative type I error rate; and a bar at the blue dashed line indicates an accurate type I error rate (i.e., $p=0.05$ ). The standard deviation of the mean type I error rate was also marked on the bar plot.

comparable because it cannot be distinguished whether a higher/lower power is a result of the test procedure itself, or because of inflated/conservative type I error rate control. Only the LR_rhythmicity and the permutation test survived these criteria. Figure S8 shows the power with respect to varying sample sizes. Both these methods are similarly powerful at $5 \%$ nominal level of type I error rate. When the sample size is larger, both tests became more powerful. However, we want to point out that the precision of the permutation test depends heavily on the number of permutations. For example, it may need at least 1,000,000 permutations in order to achieve a $p<10^{-6}$, which could be a computational burden. The LR_rhythmicity has no such restriction, and could obtain an arbitrarily small p-values without extra computational concerns.

\section{Differential Circadian Analysis}

In this section, we used simulation to evaluate the performance of the likelihood-based method in detecting differential circadian patterns, including differential amplitude, differential phase, differential basal level, and differential fit. We first compared among our proposed likelihood-based methods including Wald test (regular), Wald test (finite sample), likelihood ratio test (regular), likelihood ratio test (finite sample). We found that likelihood ratio test (finite sample) was the best performer of our proposed methods. We then compared this best performer with other existing methods for differential circadian pattern analysis, including Circacompare, limorhyde, HANOVA, robustDODR, and the permutation test under variety of simulation settings.

\section{Simulation settings}

The simulation setting is based on Equation 5. The basic parameter setting for simulation is listed as below. We set number of genes $G=10,000$ and the sample size $n$ was set to be 10 . For each gene $g(1 \leq g \leq G)$, amplitudes $A_{1}=A_{2}$ were set to be 3 ; phases $\phi_{1}=\phi_{2}$ were generated from $\operatorname{UNIF}(0,24)$. Basal levels $C_{1}=C_{2}$ were generated from $\operatorname{UNIF}(10,13)$. Error terms $\varepsilon_{i}, \varepsilon_{j}$ were generated from normal distribution $N\left(0, \sigma_{1}^{2}\right)$ and $N\left(0, \sigma_{2}^{2}\right)$, respectively. $\sigma_{1}^{2}=\sigma_{2}^{2}$ were set to be 1 . This simulation was repeated 10 times to increase numbers of replications and to obtain standard error estimate. To examine the impact of sample size, correlation between genes, and distribution violations, we further simulated the following variations.

1. Impact of sample sizes. We varied $n=10,20,50$ while fixing other parameters in the basic parameter setting.

2. Impact of correlated genes. For every $m=50$ genes, we simulated the correlated gene structure as described in Section 3.1.1. We varied the strength of correlation $\rho=0,0.25,0.5$ while fixing other parameters in the basic parameter setting.

3. Violation of the Gaussian assumption. As described in Section 3.1.1, we varied the error distribution $\varepsilon_{1}=$ $\varepsilon_{2} \sim t(3), t(5), t(10), t(\infty)$ to mimic different levels of violation of normality assumptions.

The best performer of the likelihood-based methods in detecting differential circadian patterns

We evaluated the type I error rate (nominal $\alpha$ level $5 \%$ ) of our proposed likelihood-based methods, including Wald test (regular), Wald test (finite sample), likelihood ratio test (regular), likelihood ratio test (finite sample), under all pre-mentioned simulation settings. Figure S9, S10 show that the likelihood ratio test (finite sample) had the best performance in terms of type I error rate control with varying sample size or strength of correlation among genes. Thus, we denoted this method as LR_diff, and will further compared LR_diff with other existing methods. In Figure S11, when there was a violation of the Gaussian assumption, we observed that LR_diff still controlled the type I error rate for differential amplitude, differential phase, and differential basal levels, but resulted in inflated type I error rate of differential fit. This is not unexpected since likelihood-based methods utilized the Gaussian assumption to derive the test statistics. Under this situation, we would recommend users to take transformation (i.e., log transformation) to improve normality (See Section 5 for more discussions).

Type I Error rate comparison with other methods We evaluated the type I error rate (nominal $\alpha$ level $5 \%$ ) of the following methods: LR_diff, Circacompare, limorhyde, HANOVA, robustDODR, and permutation test under different simulation settings (See Section 3.2.1 for details). Here, LR_diff and the permutation test are applicable for testing all 4 types of differential circadian analysis in Figure 1; HANOVA, robustDODR and LimoRhyde are designed to detect differential rhythmicity (i.e., whether the circadian rhythmicity across two conditions are identical), and cannot distinguish the 4 subcategories. 
Thus we will apply these three methods in detecting differential fit, which is closely related to differential rhythmicity conceptually; and Circacompare is applicable for testing differential amplitude, differential phase, and differential basal levels (Also see Table 1 for for their applicability).

1. Impact of sample sizes. Based on the basic parameter setting, we varied $n=10,20,50$. Figure 4 shows the type I error rate control for the 6 methods. Among which 3 methods were applicable for detecting differential amplitude, differential basal level, differential phase, including LR_diff, Circacompare and the permutation test. All these 3 methods could control the type I error rate to the $5 \%$ nominal level, though Circacompare is slightly better than LR_diff and the permutation test. In addition, 5 methods were applicable for detecting differential fit, including LR_diff, limorhyde, HANOVA, robustDODR, and the permutation test. We observed that LR_diff, limorhyde, and the permutation test could control the type I error rate to the $5 \%$ nominal level, while HANOVA and robustDODR may have slightly inflated type I error rate. We observed their performance did not rely heavily on the sample size, which is expected since these methods did not necessarily rely on large sample size.

2. Impact of correlated genes. Figure S12 shows the type I error rate control by varying the strength of correlations between genes. Similar to the previous simulation setting, we did not observe the correlated gene structure had a big impact on their performance.

3. Violation of the Gaussian assumption. Instead of assuming the error term was generated from a standard normal distribution (i.e., $N(0,1)$ ), we generated $\varepsilon_{1} \sim t(3), t(5), t(10), t(\infty)$, where $t(d f)$ is the t-distribution with degree of freedom $d f$. Smaller $d f$ represents longer tailed error distribution, and thus larger violation of the normality assumption. Figure S13 shows the type I error rate control for the 6 methods. In terms of differential amplitude, differential basal level, differential phase, LR_diff, Circacompare and the permutation test successfully controlled the type I error rate to the $5 \%$ nominal. In terms of differential fit, we observed that the LR_diff would obtain inflated type I error rate, while the performance of limorhyde, HANOVA, robustDODR, and the permutation test were similar regardless of violation of the Gaussian assumption. This is not unexpected because our likelihood-based method relied on the Gaussian assumption to derive its test statistics. Under this situation, we would recommend uses to take transformation (i.e., log transformation) to improve normality (See Section 5 for more discussions).

To summarize, in terms of differential amplitude, differential basal level, differential phase, LR_diff, Circacompare and the permutation test could control the type I error rate to the $5 \%$ nominal level. In terms of

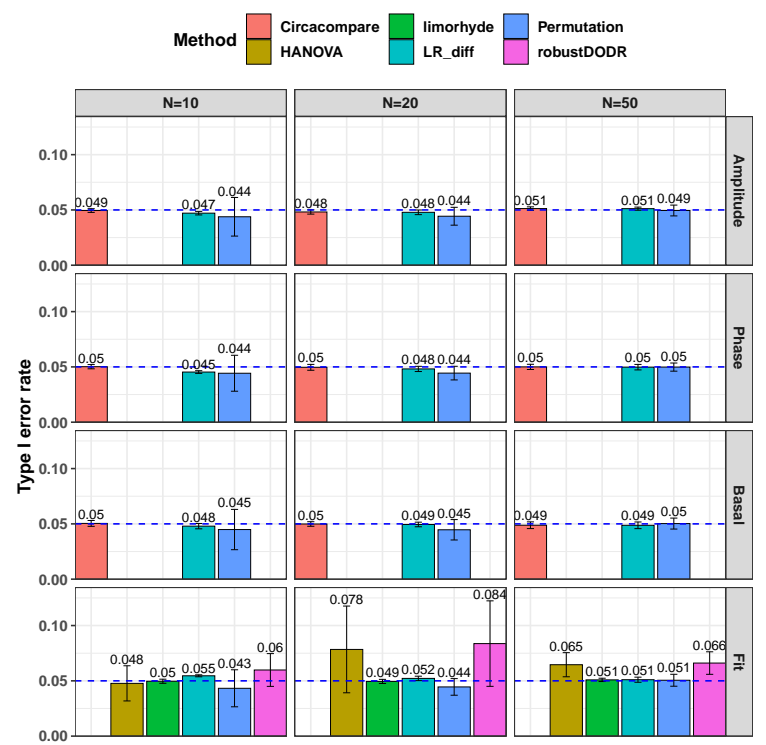

Fig. 4. Type I error rate at nominal $\alpha$ level $5 \%$ for 6 different methods in detecting differential circadian patterns. The differential circadian patterns include differential amplitude (Amplitude), differential phase (Phase), differential basal level (Basal), and differential fit (Fit). The sample sizes were varied at $\mathrm{N}=10,20$, and 50. The blue dashed line is the 5\% nominal level. A higher than $5 \%$ blue dashed line bar indicates an inflated type I error rate; a lower than $5 \%$ blue dashed line bar indicates a conservative type I error rate; and a bar at the blue dashed line indicates an accurate type I error rate (i.e., p-value $=0.05$ ). The standard deviation of the mean type I error rate was also marked on the bar plot.

differential fit and under normality assumption, LR_diff, limorhyde, and the permutation test could control the type I error rate to the 5\% nominal level, while HANOVA and robustDODR may have slightly inflated type I error rate.

Power analysis

In principle, all methods could control the type I error rate to the $5 \%$ nominal level, we included all these methods in the power evaluation (Figure 5). In terms of differential amplitude, differential basal level, differential phase, with increasing sample size or larger effect size, all three methods, including LR_diff, Circacompare, and the permutation test became more powerful. Fixing the sample size and effect size, we observed that LR_diff and Circacompare are a little bit more powerful than the permutation test. In terms of differential fit, remarkable, our proposed LR_diff is much more powerful than the permutation test, limorhyde, HANOVA, and robustDODR. In addition, with increasing sample size or larger effect size, LR_diff and the permutation test are becoming more powerful, while the other methods remained similar power or had a little bit elevated power. Table 1 summarizes and applicability, performance of type I error rate control and power for all these methods.

Remark 2 We observed that our proposed LR_diff had very similar type $I$ error rate and statistical power 


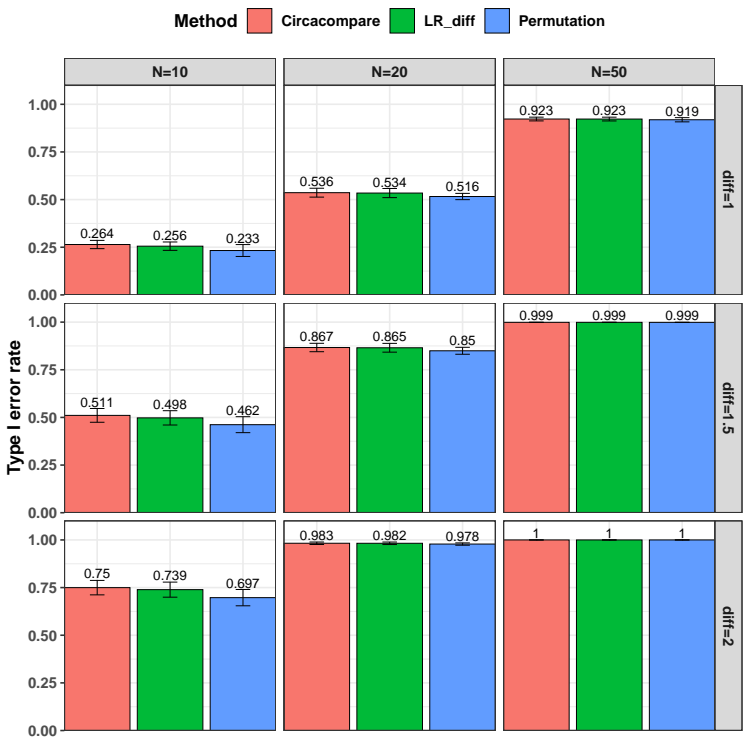

(a) Differential amplitude

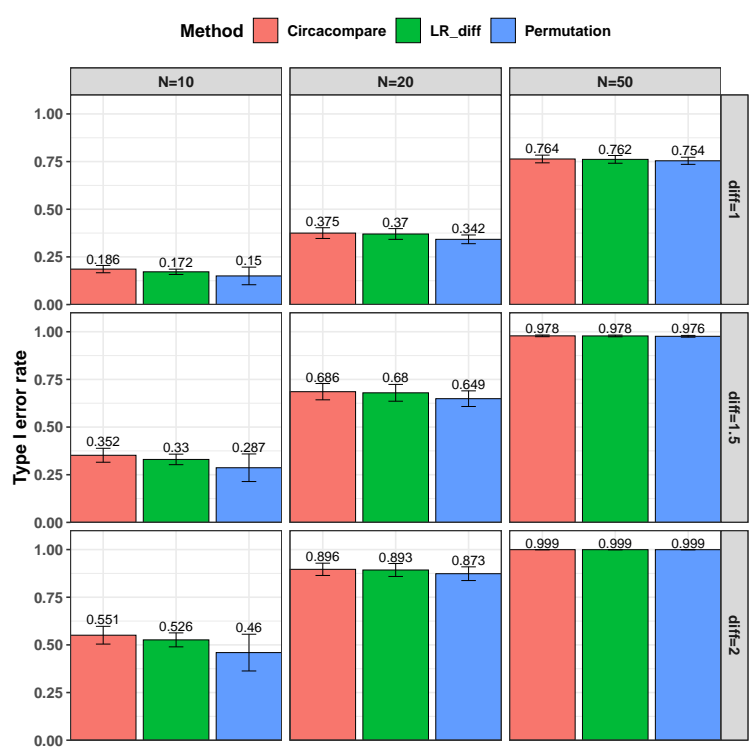

(c) Differential phase

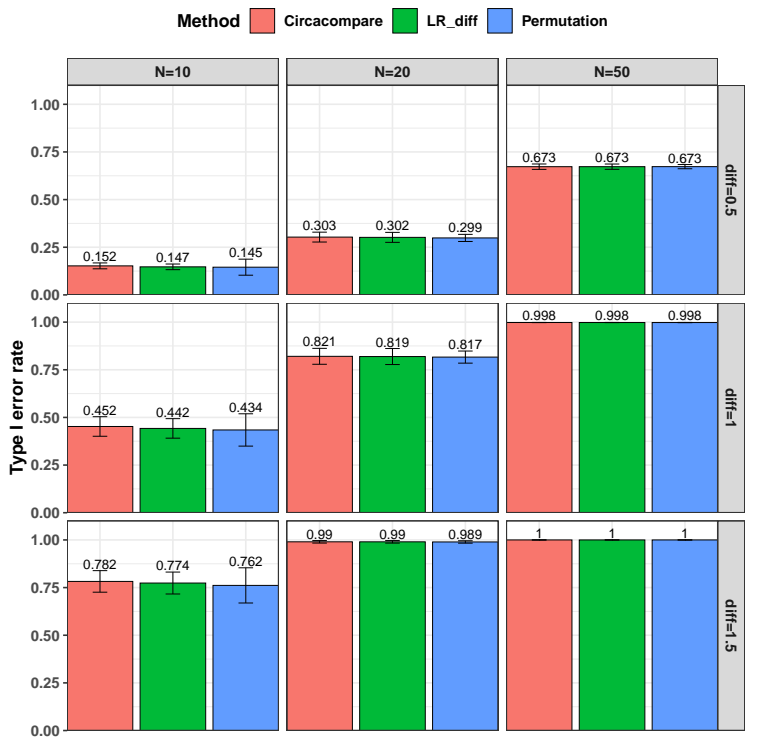

(b) Differential basal level

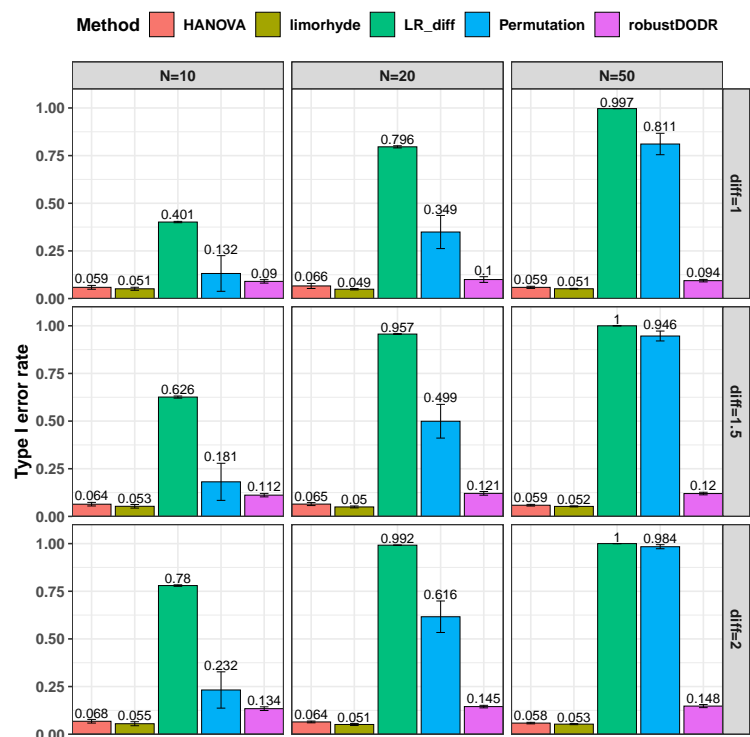

(d) Differential fit

Fig. 5. Power evaluation for 6 different methods in detecting differential circadian patterns. The differential circadian patterns include differential amplitude, differential phase, differential basal level, and differential fit. The sample sizes were varied at $\mathrm{N}=10,20$, and 50 . The standard deviation of the mean type I error rate was also marked on the bar plot.

compared to Circacompare. In fact, both LR_diff and Circacompare were designed to address the same question (i.e., differential amplitude, phase, and basal level) by deploying cosinor-based rhythmometry. The difference is that LR_diff utilized a likelihood ratio test, whereas Circacompare employed a non-linear least square approach. In addition, LR_diff is capable of testing different fit, while Circacompare cannot be used to perform this test.

\section{Real data applications}

We evaluated our likelihood-based methods (LR_rhythmicity and LR_diff) in two real data applications, including a gene expression microarray data of human postmortem brain (comparing chronological age [i.e., young v.s. old]), and a gene expression RNA sequencing data of human skeletal muscles (comparing time-restricted feeding [i.e., restricted v.s. unrestricted]). Throughout this section, we used $p \leq 0.01$ as the cutoff to declare statistical significance 
Table 1. Comparison of LR_diff with other existing methods in detecting differential circadian patterns. $\checkmark$ indicates a method is applicable; or could control the type I error to the nominal level; * indicates the most powerful method among all applicable methods. - indicates the method could roughly control the type I error to the nominal level, but with a non-negligible deviation.

\begin{tabular}{lccccc}
\hline \hline & \multicolumn{2}{c}{ Differential amp/phase/basal } & & \multicolumn{2}{c}{ Differential fit } \\
\cline { 2 - 3 } \cline { 5 - 6 } & Applicable & Type I error & & Applicable & Type I error \\
\hline LR_diff & $\checkmark$ & $\checkmark^{*}$ & & $\checkmark$ & $\checkmark^{*}$ \\
Permutation & $\checkmark$ & $\checkmark^{*}$ & & $\checkmark$ & $\checkmark$ \\
Circacompare & $\checkmark$ & $\checkmark^{*}$ & & & \\
limorhyde & & & & $\checkmark$ & $\checkmark$ \\
HANOVA & & & $\checkmark$ & - \\
robustDODR & & & $\checkmark$ & - \\
\hline \hline
\end{tabular}

unless otherwise specified. Since our likelihood-based method includes both circadian rhythmicity p-values and differential circadian pattern p-values, we denote $p_{c}$ as a p-value for circadian rhythmicity detection (i.e., from LR_rhythmicity), and $p_{d}$ as a p-value for differential circadian pattern analysis i.e., from LR_diff). $p_{d}$ can also be expanded as $p_{d}^{(a)}$ (differential amplitude); $p_{d}^{(p)}$ (differential phase); $p_{d}^{(c)}$ (differential basal level); $p_{d}^{(f)}$ (differential fit). We did not compare our methods with existing methods in the real data application, because there is no underlying truth in the real data, and thus it is difficult to benchmark their performance.

\section{Brain aging data}

We first examined our methods in a transcriptomic profile in a human postmortem brain data (Brodmann's area 11 in the prefrontal cortex). Detailed description of this study has been previously described by Chen et al. [7]. The final samples included 146 individuals whose time of death (TOD) could be precisely determined. The mean age at death was 50.7 years; $78 \%$ of the individuals were male, and the mean postmortem interval was 17.3 hours. The TODs were further adjusted as the Zeitgeber time (ZT), which adjusted factors including time zone, latitude, longitude, and altitude. The ZT was used as the circadian time, which was comparable across all individuals. 33,297 gene probes were available in this microarray data, which was publicly available in GEO (GSE71620). After filtering $50 \%$ gene probes with lower mean expression level, 16,648 gene probes were kept.

Circadian rhythmicity detection

Under $p_{c} \leq 0.01$, we detected 528 significant circadian genes using LR_rhythmicity. Figure 6 shows the 6 core circadian genes, including PER1, PER2, PER3, $A R N T L, N R 1 D 1$, and $D B P$, which are known to have persistent circadian rhythmicity. All these 6 circadian genes rendered significant p-value $\left(5.15 \times 10^{-24} \sim 3.59 \times\right.$ $10^{-6}$ ), showing the good detection power of our method in identifying circadian patterns. We further performed pathway enrichment analysis. Using pathway analysis $p \leq 0.01$ as cutoff, LR_rhythmicity detected 4 pathways. The most significant pathway was the circadian rhythm

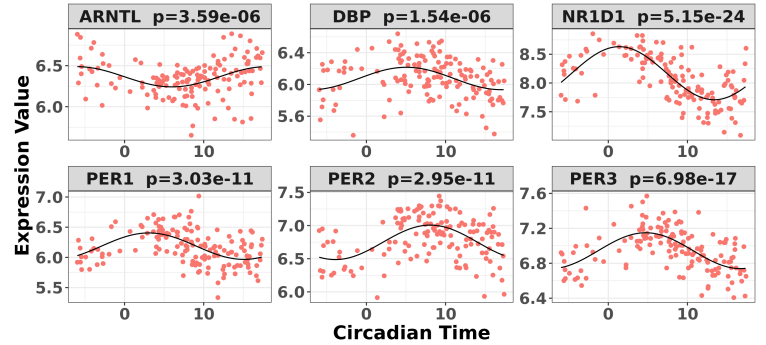

Fig. 6. Circadian rhythmicity for 6 core circadian genes in the brain aging data, including PER1, PER2, PER3, ARNTL, NR1D1, and $D B P$, using LR_rhythmicity.

signaling pathway $\left(p=3.16 \times 10^{-6}\right)$. The second most significant pathway was the senescence pathway $(p=$ $4.27 \times 10^{-4}$ ), which was also known to be associated with circadian oscillation [34].

Differential circadian analysis

In order to examine whether the chronological age was associated with disruption of circadian patterns, we further performed differential circadian analysis comparing the young group and the old group using our likelihoodbased method. We first divided the 146 individuals into two groups: young group (age $\leq 40, \mathrm{n}=31$ ) and old group (age $>60, \mathrm{n}=37$ ). Under $p_{c} \leq 0.01$, we identified 205 genes showing circadian rhythmicity in young group and 164 genes in old group, with a total of 363 unique genes, and 6 common genes.

In terms of differential fit, we started with 363 candidate genes that showed circadian rhythmicity $\left(p_{c} \leq\right.$ 0.01) in either young or old. Comparing the old group to the young group (baseline group), LR_diff identified 6 genes showing differential fit $\left(p_{d}^{(f)} \leq 0.01\right)$. As shown in Figure 1d, MYO5A is the gene showing most differential fit $\left(p_{d}^{(f)}=4.34 \times 10^{-8}\right)$, where there was circadian rhythmicity in the young group, but not in the old group. In terms of differential amplitude, differential phase, and differential basal level, we started with 6 candidate genes that showed circadian rhythmicity $\left(p_{c} \leq 0.01\right)$ in both young and old groups. Comparing the old group to the young group (baseline group), our likelihood-based method identified 1 gene showing differential amplitude $\left(p_{d}^{(a)} \leq 0.01\right), 4$ genes showing differential phase $\left(p_{d}^{(p)} \leq\right.$ $0.01), 2$ genes showing differential basal level $\left(p_{d}^{(b)} \leq 0.01\right)$. Figure 1a-1c showed the most significant genes in terms of differential amplitude (CIART, $p=0.008)$, differential phase $\left(P E R 2, p=5.43 \times 10^{-5}\right)$, and basal level (TRIB2, $\left.p=2.88 \times 10^{-6}\right)$ comparing young and old groups, respectively.

Due to the small sample size and relatively weak transcriptomic alterations in brain tissues, the number of candidate genes for differential circadian analysis was small. Thus we further relaxed the criteria to be $p_{c} \leq$ 0.05 , and we identified 897 rhythmic genes in the young group and 846 rhythmic genes in the old group. In terms of differential fit, among 1,688 genes that showed circadian rhythmicity $\left(p_{c} \leq 0.05\right)$ in either young or 
old group, LR_diff identified 345 genes showing gain or loss of rhythmicity. In terms of differential amplitude, differential phase, and differential basal level, we started with 55 candidate genes that showed circadian rhythmicity $\left(p_{c} \leq 0.05\right)$ in both young and old groups. Comparing the old group to the young group (baseline group), LR_diff identified 2 genes showing differential amplitude, 23 genes showing differential phase, 19 genes showing differential basal level.

\section{Time-restricted feeding data}

We evaluated the performance of our likelihood-based methods in transcriptomic profiles of skeletal muscle tissue. 11 overweight or obese men were included in this dataset; the age range was 30-45 years; the body mass index [BMI] range was $27-35 \mathrm{~kg} / \mathrm{m}^{2}$. These participants were randomized into time-restricted feeding (TRF) group and the un-restricted feeding (URF) group by adopting a cross over design, where each participant was assigned to both TRF and URF groups in different time periods. The skeletal muscle samples of each participant under each experimental group were repeatedly measured every 4 hours over 24 hours. There were some missing measurement, but each participant had $4 \sim$ 6 measurement, resulting in a total of 63 samples in restricted group and 62 samples in unrestricted group. Detailed description of this study has been previously published [35]. This RNA-seq dataset is publicly available in GEO (GES129843). After filtering the gene probes with mean cpm less than 1, 13,167 gene probes were kept. We further performed $\log 2$ transformation [i.e., $\log 2(x+1)$, where $x$ is the cpm of a gene in a sample] to improve the normality of the data.

Circadian pattern detection

We first applied the LR_rhythmicity method to this time-restricted feeding dataset. Under $p_{c} \leq 0.01$, we identified 1,407 and 935 genes showing significant circadian rhythmicity for the restricted group and the unrestricted group, respectively. Figure S14 and S15 shows the 6 core circadian genes in the TRF group and the URF group, including PER1, PER2, PER3, ARNTL, $N R 1 D 1$, and $D B P$, which are known to have persistent circadian rhythmicity. For these 6 circadian genes for the restricted group and the unrestricted group, our method (LR_rhythmicity) yielded highly significant p-value $(1.35 \times$ $\left.10^{-8} \sim 1.65 \times 10^{-21}\right)$, showing the strong detection power of circadian rhythmicity. We further performed pathway enrichment analysis. Using pathway $\leq 0.01$ as cutoff, our likelihood methods detected 61 and 105 significant pathways for the TRF group and URF group respectively. The top pathways enriched in both groups included Circadian Rhythm Signaling pathway, Prolactin Signaling pathway, IGF-1 Signaling pathway, which are all known to related with circadian rhythmicity $[36,37]$.

Differential circadian analysis

We further performed differential circadian analysis comparing TRF and URF groups using LR_diff. In terms of differential fit, we started with candidate genes that showed circadian rhythmicity $\left(p_{c} \leq 0.01\right)$ in either restricted or unrestricted $(n=1,864)$. Comparing the TRF group to the URF group (baseline group), LR_diff identified 57 genes showing differential fit $\left(p_{d}^{(f)} \leq\right.$ $0.01)$. The most significant gene, $R U F Y 1$, is shown in Figure S16(d), where there was a rhythmicity in the TRF group but not in the URF group.

In terms of differential amplitude, differential phase, and differential basal level, we started with candidate genes that showed circadian rhythmicity $\left(p_{c} \leq 0.01\right)$ in both TRF and URF $(n=478)$. Comparing TRF to URF, 11 genes showing differential amplitude $\left(p_{d}^{(a)} \leq\right.$ $0.01), 25$ genes showing differential phase $p_{d}^{(p)} \leq 0.01$, 8 genes showing differential basal level $p_{d}^{(b)} \leq 0.01$. Figure S16(a-c) showed the most significant genes for differential amplitude, phase, and basal level comparing TRF and URF groups, respectively.

\section{Discussion}

In summary, we developed a series of likelihood-based methods for detecting (i) circadian rhythmicity and (ii) differential circadian patterns. In terms of circadian rhythmicity detection, our method (LR_rhythmicity) could better control the type I error rate to its nominal level (i.e., produce an accurate p-values) than the other competing methods. In terms of differential circadian patterns, our likelihood-based method is the first parametric method to characterize 4 sub-categories of differential circadian patterns, including differential amplitude, differential phase, differential basal level, and differential fit. Simulation shows that our method (LR_diff) successfully controlled the type I error rate to the $5 \%$ nominal level for all 4 types of differential circadian patterns under the Gaussian assumption. In addition, LR_diff was more powerful than the competing methods in terms of differential fit. We also applied our methods in transcriptomic data applications including a brain aging data, and a time restricted feeding data. Superior performance has been observed in these applications.

Our methods have the following strengths. (i) The type I error rate of both LR_rhythmicity and LR_diff were well controlled, indicating the p-values from these methods are accurate. While in the literature, it remained a concern about the type I error rate control for existing methods in terms of detecting circadian rhythmicity. (ii) Some methods require integer input circadian time, and even intervals between adjacent circadian time, while our methods have no such restrictions. Circadian time from modern epidemiology studies usually unevenly distributed between 0 hours and 24 hours. Thus, our method can be more applicable in biomedical applications. (iii) For examining differential fit, our method is statistically more powerful compared to other existing methods.

Our methods could potentially suffer from the following limitation. Our proposed methods are based on likelihood, which assume the residuals are normally distributed. And as shown in our simulation, the violation of such Gaussian assumptions may result in inflated type I error rate. To address this issue, we would recommend users to 
check the normality assumptions of the residuals. If the residuals (i.e., $y_{i}-\hat{y}_{i}$ ) violated the Gaussian assumption, we would recommend to take transformation (i.e., log transformation) of the raw data to improve normality. We have adopted this approach for the dietary restriction real data example.

We plan to do the following future works. (i) In epidemiology studies, many other biological factors (e.g., age, gender, etc) could have a confounding impact on the circadian rhythmicity. Thus we will enable adjusting for covariates under our likelihood-based framework. (ii) To the best of our knowledge, no circadian rhythmicity detection method could handle repeated measurement from the same individuals. For example, the time restricted feeding data example employed a cross-over design, and the 11 participants with each participant repeatedly measured 4-6 times. By extending our methods to model this within subject correlation, we would expect higher power to detect circadian rhythmicity and differential circadian patterns.

An $\mathrm{R}$ package for our method is publicly available on GitHub https://github.com/diffCircadian/diffCircadian.

\section{Competing interests}

There is NO Competing Interest.

\section{Acknowledgments}

We thank the anonymous reviewers for their valuable suggestions.

\section{Funding}

H.D., L.M., K.E., Z.H. are supported by R01HL153042; C.M. and G.T. are supported by R01MH111601.

\section{References}

1. Pietro Badia, Brent Myers, Maren Boecker, Janice Culpepper, and JR Harsh. Bright light effects on body temperature, alertness, eeg and behavior. Physiology Es behavior, 50(3):583-588, 1991.

2. Christopher M Jung, Sat Bir S Khalsa, Frank AJL Scheer, Christian Cajochen, Steven W Lockley, Charles A Czeisler, and Kenneth P Wright Jr. Acute effects of bright light exposure on cortisol levels. Journal of biological rhythms, 25(3):208-216, 2010.

3. Angelo Cagnacci, JA Elliott, and SS Yen. Melatonin: a major regulator of the circadian rhythm of core temperature in humans. The Journal of Clinical Endocrinology \& Metabolism, 75(2):447-452, 1992.

4. DERK-JAN DIJK, Jeanne F Duffy, and Charles A Czeisler. Circadian and sleep/wake dependent aspects of subjective alertness and cognitive performance. Journal of sleep research, 1(2):112-117, 1992.

5. Jennifer M Hurley, Jennifer J Loros, and Jay C Dunlap. Circadian oscillators: Around the transcription-translation feedback loop and on to output. Trends in biochemical sciences, 41(10):834-846, 2016.

6. MH Hastings, M Brancaccio, and ES Maywood. Circadian pacemaking in cells and circuits of the suprachiasmatic nucleus. Journal of neuroendocrinology, 26(1):2-10, 2014.

7. Cho-Yi Chen, Ryan W Logan, Tianzhou Ma, David A Lewis, George C Tseng, Etienne Sibille, and Colleen A McClung. Effects of aging on circadian patterns of gene expression in the human prefrontal cortex. Proceedings of the National Academy of Sciences, 113(1):206-211, 2016.

8. Marianne L Seney, Kelly Cahill, John F Enwright, Ryan W Logan, Zhiguang Huo, Wei Zong, George Tseng, and Colleen A McClung. Diurnal rhythms in gene expression in the prefrontal cortex in schizophrenia. Nature communications, 10(1):1-11, 2019.

9. Brian A Hodge, Yuan Wen, Lance A Riley, Xiping Zhang, Jonathan H England, Brianna D Harfmann, Elizabeth A Schroder, and Karyn A Esser. The endogenous molecular clock orchestrates the temporal separation of substrate metabolism in skeletal muscle. Skeletal muscle, 5(1):17, 2015.

10. Michael E Hughes, Luciano DiTacchio, Kevin R Hayes, Christopher Vollmers, S Pulivarthy, Julie E Baggs, Satchidananda Panda, and John B Hogenesch. Harmonics of circadian gene transcription in mammals. PLoS Genet, 5(4):e1000442, 2009.

11. Carla S Möller-Levet, Simon N Archer, Giselda Bucca, Emma E Laing, Ana Slak, Renata Kabiljo, June CY Lo, Nayantara Santhi, Malcolm von Schantz, Colin P Smith, et al. Effects of insufficient sleep on circadian rhythmicity and expression amplitude of the human blood transcriptome. Proceedings of the National Academy of Sciences, 110(12):E1132-E1141, 2013.

12. Ray Zhang, Nicholas F Lahens, Heather I Ballance, Michael E Hughes, and John B Hogenesch. A circadian gene expression atlas in mammals: implications for biology and medicine. Proceedings of the National Academy of Sciences, 111(45):16219-16224, 2014.

13. Marc D Ruben, Gang Wu, David F Smith, Robert E Schmidt, Lauren J Francey, Yin Yeng Lee, Ron C Anafi, and John B Hogenesch. A database of tissue-specific rhythmically expressed human genes has potential applications in circadian medicine. Science Translational Medicine, 10(458), 2018.

14. Dirk Jan Stenvers, Aldo Jongejan, Sadaf Atiqi, Jeroen P Vreijling, Eelkje J Limonard, Erik Endert, Frank Baas, Perry D Moerland, Eric Fliers, Andries Kalsbeek, et al. Diurnal rhythms in the white adipose tissue transcriptome are disturbed in obese individuals with type 2 diabetes compared with lean control individuals. Diabetologia, 62(4):704-716, 2019.

15. Jun Z Li, Blynn G Bunney, Fan Meng, Megan H Hagenauer, David M Walsh, Marquis $\mathrm{P}$ Vawter, Simon J Evans, Prabhakara V Choudary, Preston Cartagena, Jack D Barchas, et al. Circadian patterns of gene expression in the human brain and disruption in major depressive disorder. Proceedings of the National Academy of Sciences, 110(24):9950-9955, 
2013.

16. Andrew SP Lim, Hans-Ulrich Klein, Lei Yu, Lori B Chibnik, Sanam Ali, Jishu Xu, David A Bennett, and Philip L De Jager. Diurnal and seasonal molecular rhythms in human neocortex and their relation to alzheimer's disease. Nature communications, 8(1):116, 2017.

17. Germaine Cornelissen. Cosinor-based rhythmometry. Theoretical Biology and Medical Modelling, 11(1):16, 2014.

18. Earl F Glynn, Jie Chen, and Arcady R Mushegian. Detecting periodic patterns in unevenly spaced gene expression time series using lomb-scargle periodograms. Bioinformatics, 22(3):310-316, 2006.

19. Martin Straume. Dna microarray time series analysis: automated statistical assessment of circadian rhythms in gene expression patterning. In Methods in enzymology, volume 383, pages 149-166. Elsevier, 2004 .

20. Rendong Yang and Zhen Su. Analyzing circadian expression data by harmonic regression based on autoregressive spectral estimation. Bioinformatics, 26(12):i168-i174, 2010.

21. Paul F Thaben and Pål O Westermark. Detecting rhythms in time series with rain. Journal of biological rhythms, 29(6):391-400, 2014.

22. Michael E Hughes, John B Hogenesch, and Karl Kornacker. Jtk_cycle: an efficient nonparametric algorithm for detecting rhythmic components in genome-scale data sets. Journal of biological rhythms, 25(5):372-380, 2010.

23. Gang Wu, Ron C Anafi, Michael E Hughes, Karl Kornacker, and John B Hogenesch. Metacycle: an integrated $\mathrm{r}$ package to evaluate periodicity in large scale data. Bioinformatics, 32(21):3351-3353, 2016.

24. Michael E Hughes, Katherine C Abruzzi, Ravi Allada, Ron Anafi, Alaaddin Bulak Arpat, Gad Asher, Pierre Baldi, Charissa De Bekker, Deborah Bell-Pedersen, Justin Blau, et al. Guidelines for genome-scale analysis of biological rhythms. Journal of biological rhythms, 32(5):380-393, 2017.

25. David Laloum and Marc Robinson-Rechavi. Methods detecting rhythmic gene expression are biologically relevant only for strong signal. PLoS computational biology, 16(3):e1007666, 2020.

26. Wenwen Mei, Zhiwen Jiang, Yang Chen, Li Chen, Aziz Sancar, and Yuchao Jiang. Genome-wide circadian rhythm detection methods: systematic evaluations and practical guidelines. bioRxiv, 2020.

27. Jacob J Hughey and Atul J Butte. Differential phasing between circadian clocks in the brain and peripheral organs in humans. Journal of biological rhythms,
31(6):588-597, 2016.

28. Polly Yingshan Hsu and Stacey L Harmer. Circadian phase has profound effects on differential expression analysis. PloS one, 7(11):e49853, 2012.

29. Anne Pelikan, Hanspeter Herzel, Achim Kramer, and Bharath Ananthasubramaniam. Studies overestimate the extent of circadian rhythm reprogramming in response to dietary and genetic changes. bioRxiv, 2020.

30. Paul F Thaben and Pål O Westermark. Differential rhythmicity: detecting altered rhythmicity in biological data. Bioinformatics, 32(18):2800-2808, 2016.

31. Jordan M Singer and Jacob J Hughey. Limorhyde: a flexible approach for differential analysis of rhythmic transcriptome data. Journal of biological rhythms, 34(1):5-18, 2019.

32. Rex Parsons, Richard Parsons, Nicholas Garner, Henrik Oster, and Oliver Rawashdeh. Circacompare: a method to estimate and statistically support differences in mesor, amplitude and phase, between circadian rhythms. Bioinformatics, 36(4):1208-1212, 2020.

33. Thomas Parker. Finite-sample distributions of the wald, likelihood ratio, and lagrange multiplier test statistics in the classical linear model. Communications in Statistics - Theory and Methods, 46(11):5195-5202, 2017.

34. Takeshige Kunieda, Tohru Minamino, Taro Katsuno, Kaoru Tateno, Jun-ichiro Nishi, Hideyuki Miyauchi, Masayuki Orimo, Sho Okada, and Issei Komuro. Cellular senescence impairs circadian expression of clock genes in vitro and in vivo. Circulation research, 98(4):532-539, 2006.

35. Leonidas S Lundell, Evelyn B Parr, Brooke L Devlin, Lars R Ingerslev, Ali Altıntaş, Shogo Sato, Paolo Sassone-Corsi, Romain Barrès, Juleen R Zierath, and John A Hawley. Time-restricted feeding alters lipid and amino acid metabolite rhythmicity without perturbing clock gene expression. Nature communications, 11(1):1-11, 2020.

36. Christopher J Morris, Daniel Aeschbach, and Frank AJL Scheer. Circadian system, sleep and endocrinology. Molecular and cellular endocrinology, 349(1):91-104, 2012.

37. Priya Crosby, Ryan Hamnett, Marrit Putker, Nathaniel P Hoyle, Martin Reed, Carolyn J Karam, Elizabeth S Maywood, Alessandra Stangherlin, Johanna E Chesham, Edward A Hayter, et al. Insulin/igf-1 drives period synthesis to entrain circadian rhythms with feeding time. Cell, 177(4):896909, 2019. 